\title{
Thermal stability of the microstructure of severely deformed copper
}

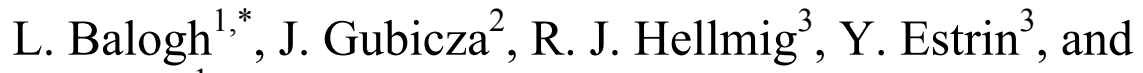 T. Ungár ${ }^{1}$}

${ }^{1}$ Department of General Physics, Eötvös University, Budapest, Hungary

${ }^{2}$ Department of Solid State Physics, Eötvös University, Budapest, Hungary

${ }^{3}$ Institute for Materials Engineering and Technology, Clausthal University of Technology, Clausthal, Germany

*Contact author; e-mail: balevi@metal.elte.hu

Keywords: copper, equal channel angular pressing, X-ray diffraction, dislocation, bimodal structure

\begin{abstract}
Copper specimens were deformed by equal channel angular pressing (ECAP) up to 8 passes. The microstructure was studied by X-ray line profile analysis. The crystallite size is reduced to a few tens of nanometers even after the first ECAP pass and it does not change significantly during further deformation. At the same time, the dislocation density increases gradually up to 4 ECAP passes. The thermal stability of the microstructure is examined by differential scanning calorimetry (DSC). The temperature of the DSC peak decreases whereas the stored energy increases with increasing strain. At the beginning of the heat release a bimodal grain structure develops indicated by a special double-peak shape of the diffraction line profiles.
\end{abstract}

\section{Introduction}

Bulk ultrafine grained metals can be produced by severe plastic deformation (SPD) $[1,2]$. If the conditions (e.g. temperature, dimensions of the sample) of SPD methods are selected properly, the manufactured specimens meet the following requirements [2]: i) high angle grain boundaries dominate in the ultrafine grained structure, ii) the microstructure is uniform within the whole volume of the specimen and iii) the materials have no cracks or mechanical damage. One of the most common SPD methods is equal channel angular pressing (ECAP). This method provides ultrafine grained metal billets with homogeneous microstructure. A billet can be deformed repeatedly using this method as the shape of the specimen does not change during ECAP deformation.

The microstructure of the ultrafine grained materials can be effectively studied by X-ray diffraction peak profile analysis. The broadening of a profile is caused by size and strain effects simultaneously. In deformed metals the lattice distortions are mainly caused by dislocations, so the strain broadening can be expressed in terms of the parameters of the dislocation structure $[3,4]$. The anisotropic strain broadening is taken into account by the contrast 
factors of the dislocations [4,5]. The dislocation structure can be determined by the evaluation of the X-ray profiles for the contrast factors because their values depend on the dislocation slip systems present in the crystal. Developments in computing enable procedures to be elaborated for determining the characteristic parameters of the microstructure by fitting the whole diffraction profiles [6-9]. In the present work the Multiple Whole Profile (MWP) fitting method is used in which the measured intensity profiles are fitted by theoretical functions calculated on the basis of the model of microstructure [8,9]. This procedure enables the determination of both the crystallite size distribution and the dislocation structure in ultrafine grained materials.

In this paper the microstructure of severely deformed copper is studied as a function of strain up to $\varepsilon=9.2$. The high strain values are achieved by the ECAP technique. The thermal stability of the ultrafine grained microstructure is also investigated. The characteristic parameters of the crystallite size distribution and the dislocation structure are determined by X-ray line profile analysis.

\section{Experimental}

Technical purity copper specimens $(99.99 \%)$ were annealed at $450{ }^{0} \mathrm{C}$ for 2 hours prior to ECA pressing to obtain a defined initial state. They were subsequently deformed by $1,2,4$ or 8 ECAP passes using a $90^{\circ}$ die following route $\mathrm{C}$ (the billet after each pass is rotated by $180^{\circ}$ around its longitudinal axis). Each pass corresponds to an equivalent strain of $\varepsilon=1.15$. This means that the samples were deformed for strain values of 1.15, 2.3, 4.6 and 9.2. An additional specimen has been deformed for $\varepsilon=0.7$ by compression using an MTS $^{\mathrm{TM}}$ hydraulic machine. Thermal stability of the ultrafine grained microstructure was investigated by differencial scanning calorimetry (DSC) using a Perkin-Elmer ${ }^{\mathrm{TM}} \mathrm{DSC} 2$ calorimeter.

The microstructures of the deformed and the heat-treated specimens were investigated by $\mathrm{X}$ ray peak profile analysis. The X-ray diffraction profiles were measured on the cross section of the billets. The measurements were performed by a double crystal diffractometer (Noni$u^{\mathrm{TM}}$ FR591) with negligible instrumental broadening using $\mathrm{CuK} \alpha_{1}$ radiation $(\lambda=0.15406$ $\mathrm{nm})$. The intensity profiles were recorded by a linear position sensitive gas-flow detector $\left(\mathrm{OED}^{\mathrm{TM}} 50\right.$ Braun, Munich). The measured data were evaluated by the MWP fitting method. The procedure is described in detail in references $[8,9]$. The measured intensity profiles are fitted by theoretical line profiles and from the procedure the following parameters of the microstructure are obtained: (i) the median $m$ and the variance $\sigma$ of the log-normal size distribution function, (ii) the density $\rho$ and the arrangement parameter $M$ of dislocations, (iii) the $q$ parameter, which characterizes the contrast factors of dislocations and describes the type of dislocations (edge or screw). The arithmetic, area- and volume-weighted mean crystallite sizes can be calculated from $m$ and $\sigma$ using formulas given in reference [6]. In this paper only the area-weighted mean crystallite size, $\langle x\rangle{ }_{\text {area }}$ is presented. The magnitude of $M$ gives the strength of the dipole character of dislocations: a higher $M$ value corresponds to a weaker dipole character and weaker screening of the displacement fields of dislocations. 


\section{Results and discussion}

\section{The microstructure of copper as a function of strain}

The area-weighted mean crystallite size has a value of $62 \pm 7 \mathrm{~nm}$ even after a single ECAP pass. The crystallite size does not change significantly by further ECAP deformation. At the same time the dislocation density increases from $8 \times 10^{14} \mathrm{~m}^{-2}$ to $28 \times 10^{14} \mathrm{~m}^{-2}$ when the number of ECAP passes increases from 1 to 4 . The dislocation density does not change significantly with further deformation. The experimental value of the $q$ parameter is $2.0 \pm 0.1$ for all samples. The $q$ parameter values for copper corresponding to pure screw and edge dislocations in the $<110>\{111\}$ slip system were determined by detailed numerical calculations using the equations from [10] and the elastic constants from [11]. This yielded the $q$ values of 2.4 or 1.7 for pure screw or edge dislocations, respectively. The experimental value of $q$ for ECA pressed $\mathrm{Cu}$ agrees well with the arithmetic average of the values calculated for pure edge and screw dislocations, which suggests that the character of dislocations is half edge half screw. The $M$ parameter decreases with increasing strain up to 4 ECAP passes. Its value is 2.2 at $\varepsilon=0.7$ and 1.1 at $\varepsilon=4.6$. For higher strains $M$ remains almost constant. The decrease of the value of $M$ indicates that with increasing strain the dipole character of the dislocation structure becomes stronger. It was established that after 4 ECAP passes there are no further changes in the microstructure.

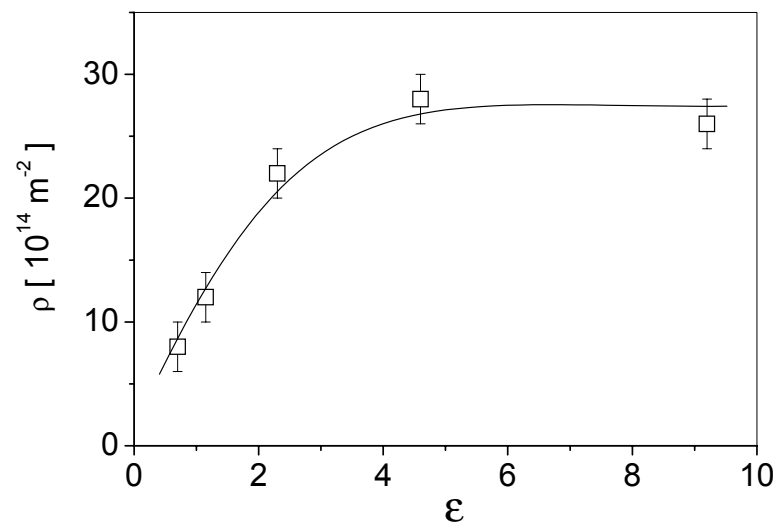

Figure 1. The dislocation density ( $\rho$ ) as a function of strain.

\section{Thermal stability of the microstructure}

The deformed copper specimens were subjected to DSC scans from $300 \mathrm{~K}$ to $1000 \mathrm{~K}$ at a heating rate of $40 \mathrm{~K} / \mathrm{min}$. For each sample a broad exothermic peak was observed on the DSC curve which corresponds to the release of the stored strain energy during the recovery of the microstructure. The maximum of the peak and the evolved heat as a function of strain are shown in figures 2 and 3, respectively. The maximum of the exothermic peak in the DSC curve shifted to lower temperature values and the heat released during the heat-treatment increased with increasing of deformation up to $\varepsilon=2.3$. These changes can be explained by the 
rise of the density and the stronger dipole character of dislocations with increasing strain. The dislocation density vs. strain (figure 1) and the stored energy vs. strain (figure 1) relations are similar, because the dislocations are the main source of strains in SPD materials. The slight difference in the strains of saturation for the two quantities can be explained by the reduction of the strain energy with the stronger dipole character of dislocation arrangement.

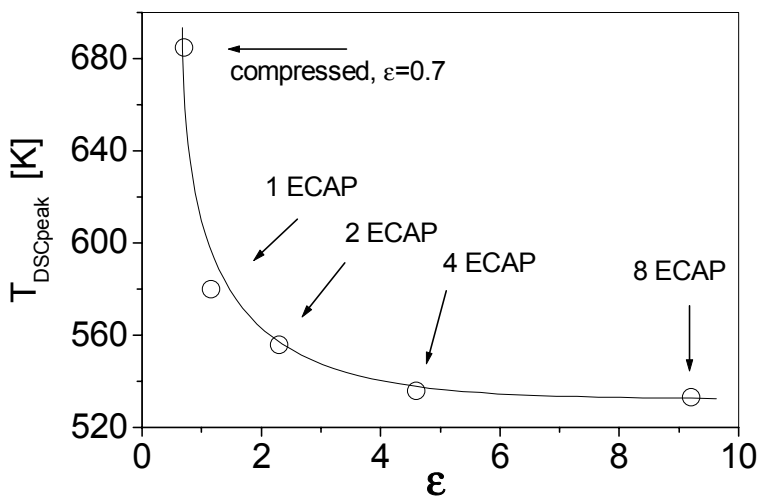

Figure 2. The temperature of the exothermic DSC peak as a function of strain.

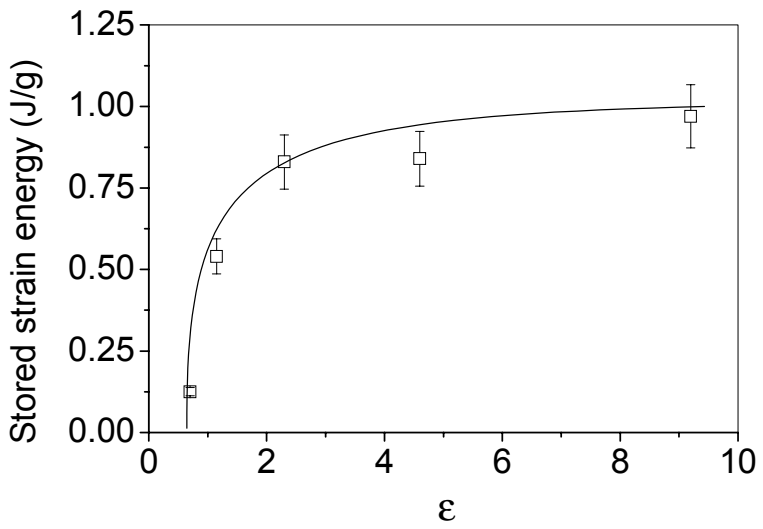

Figure 3. The stored energy released during the recovery of the microstructure as a function of strain. This energy was determined as the area of the exothermic peak in the DSC scan.

The recovery of the microstructure was studied for $\varepsilon=0.7$, after 1 ECAP and 8 ECAP passes. The specimens were heated at $40 \mathrm{~K} / \mathrm{min}$ and when the desired temperature was reached, they were quenched at $200 \mathrm{~K} / \mathrm{min}$. The temperatures were chosen before the DSC peak, at the top 
of the peak and one after the peak. Figure 4 shows the evolution of the area-weighted mean crystallite size and the dislocation density as a function of temperature in the case of the 8 ECAP specimen. The other two samples show almost identical behaviour, only the values of the temperatures and of dislocation densities being different.

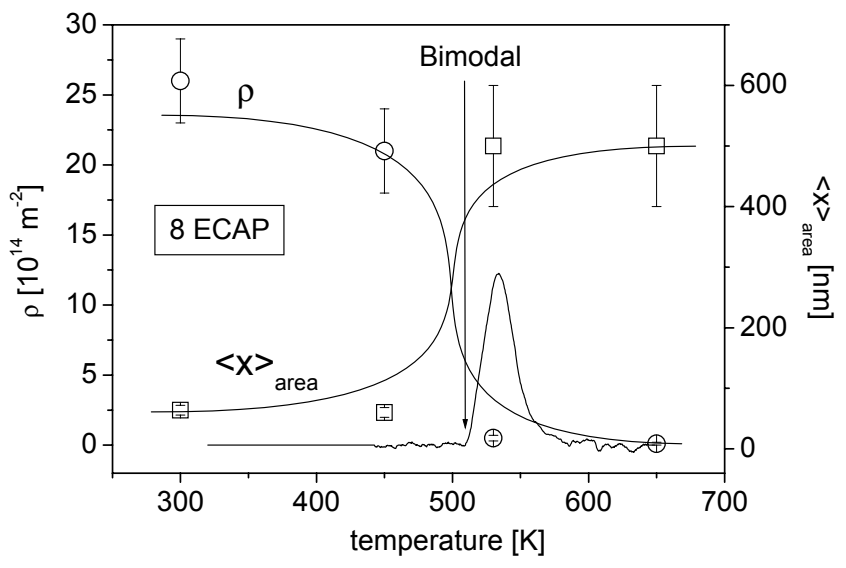

Figure 4. The area-weighted mean crystallite size and the dislocation density as a function of temperature for the sample deformed by 8 ECAP passes.

At a temperature corresponding to the beginning of the DSC peaks, none of the peaks could be fitted by a single theoretical profile and each peak seems to be the sum of a narrow and a broad peak. This special shape of the diffraction peaks has been observed previously by Kuzel et al. [12] for a copper sample deformed by torsion under 6 GPa pressure and annealed at $250{ }^{\circ} \mathrm{C}$ for 100 minutes. Figure 5 shows the 311 peak for the specimen quenched from $500 \mathrm{~K}$ (dashed line), from which it can be seen that indeed the diffraction profile does consist of two peaks. It was found that the broad peak can be well approximated by the profile measured before the heat-treatment, which indicates that the broad subprofile corresponds to the non-recovered volumes of the material. The profile for the non annealed specimen (the dotted line in figure 5) matches perfectly the tail part of the peak recorded after annealing (the dashed line in figure 5) after dividing the intensity by an appropriate factor. The difference between the two profiles (the solid line in figure 5) gives a sharp peak which corresponds to the recovered volume of the material.

\section{Conclusions}

The microstructure of severely deformed copper specimens was investigated by X-ray peak profile analysis. It was found that the crystallite size is reduced to about $60 \mathrm{~nm}$ even at $\varepsilon=0.7$ and further refinement can not be achieved by increasing strain. At the same time the dislocation density increases and the dipole character of dislocations becomes stronger with increasing strain up to $\varepsilon=4.6$. These changes in the dislocation structure result in the decrease 
of the temperature of recovery and an increase in the stored energy released during annealing. The recovery starts inhomogeneously forming bimodal microstructure in the sample.

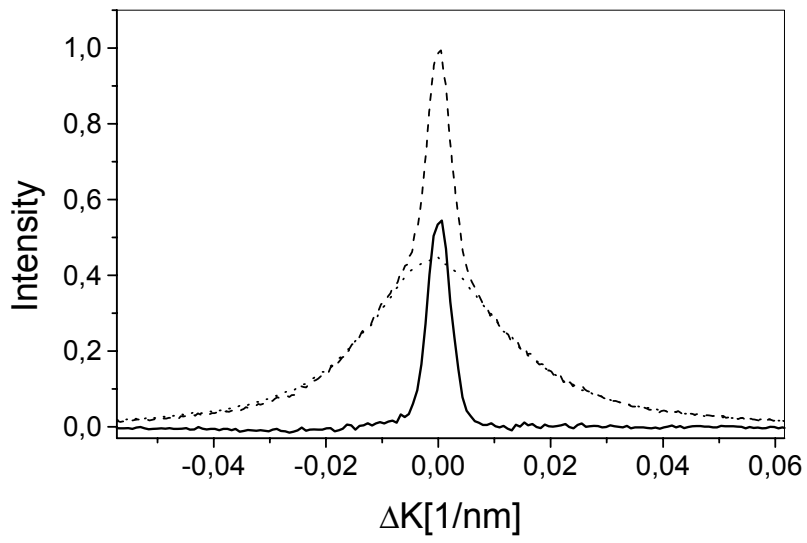

Figure 5. The difference between the 311 peak for copper deformed by 8 ECAP passes and quenched from $500 \mathrm{~K}$ (dashed line) and that of the non-annealed sample (dotted line) gives a sharp peak (solid line).

\section{References}

1. Baik, S.C., Estrin, Y., Kim, H.S., Jeong H.T. \& Hellmig, R.J., 2002, Mater. Sci. Forum 408, 697.

2. Stolyarov, V.V., Zhu, Y.T., Alexandrov, I.V., Lowe T.C. \& Valiev, R.Z., 2003, Materials Science and Engineering A 343, 43.

3. Krivoglaz, M.A., 1996, Theory of X-ray and Thermal Neutron Scattering by Real Crystals, Plenum Press, New York

4. Wilkens, M., Phys. Stat. Sol., 1970, (a) 2, 359

5. Kuzel, R. \& Klimanek, P., 1988, J. Appl. Cryst. 21, 363

6. Langford, J.I., Louer, D. \& Scardi, P., 2000, J. Appl. Cryst. 33, 964

7. Scardi, P. \& Leoni, M., 2002, Acta Cryst. A 58, 190

8. Ungár, T., Gubicza, J., Ribárik, G. \& Borbély, A., 2001, J. Appl. Cryst. 34, 298.

9. Ribárik, G., Ungár, T. \& Gubicza, J., 2001, J. Appl. Cryst. 34, 669.

10. Ungár, T., Dragomir I., Révész, Á. \& Borbély, 1999, A., J. Appl. Cryst. 32, 992

11. Hearmon, R.F.S., 1966, Landolt-Börnstein 1, 1

12. Kuzel, R., Holy, V., Cernansky, M., Kubena, J., Simek, D. \& Kub, J., 2004, Diffraction analysis of the microstructure of materials, edited by E. J. Mittemeijer \& P. Scardi (Berlin: Springer) pp. 229-245.

Acknowledgements: This research was supported by the Hungarian National Scientific Research Fund (OTKA) under contract numbers T-046990, T-043247 and F-047057. 